\title{
Serum Soluble Endoglin Versus Serum Placental Growth Factor for Early Prediction of Preeclampsia
}

\author{
Original \\ Article \\ Ali A. Bendary ${ }^{1}$, Mohamed A. Elgazzar ${ }^{1}$, Lina A. Mohammed ${ }^{2}$ \\ ${ }^{1}$ Department of Obstetrics and Gynecology, ${ }^{2}$ Department of Medical Biochemistry, \\ Faculty of Medicine, Benha University, Egypt
}

\begin{abstract}
Aim: Evaluation of the predictive ability of serum soluble endoglin (sEng) and placental growth factor (PLGF) levels estimated at the $12^{\text {th }}$ gestational week $(\mathrm{GW})$ for discrimination of women liable to develop preeclampsia (PE).

Materials and Methods: 102 PE women were diagnosed according to the American Society of Hypertension and categorized according to guidelines of American College of Obstetricians and Gynecologists. The severity of PE was judged by the difference between blood pressure $(\triangle \mathrm{BP})$ measures at time of PE diagnosis and at time of enrolment. Blood samples were obtained at the $12^{\text {th }} \mathrm{GW}$ for ELISA estimation of serum sEng and PLGF. Study outcomes included the predictive ability of these markers for development of PE and the relation between age, body mass index (BMI) and serum levels of studied biomarkers and $\triangle \mathrm{SBP}$ and $\triangle \mathrm{DBP}$.

Results: 29 and 73 women developed early- and late-onset PE, respectively and 18 women had severe, while 84 women had mild PE. At time of PE diagnosis, BP measures were increased significantly in PE women in comparison to enrolment measures and to control measures. Serum levels of sEng were significantly higher, while serum PLGF levels were significantly lower in PE women than in controls. Development of PE was positively correlated with serum sEng, while was negatively correlated with serum PLGF levels. Also, there was positive significant correlation between $\triangle \mathrm{BP}$ and BMI and serum levels of sEng, and negative significant correlation with at enrolment BP and serum levels of PLGF. ROC curve analysis defined $\triangle \mathrm{SBP}$ and $\triangle \mathrm{DBP}$ by 33 and $10 \mathrm{mmHg}$ as a cutoff point for diagnosis of PE and defined high serum sEng as the significant sensitive predictor for development of PE at both cutoff points.

Conclusion: At $12^{\text {th }} \mathrm{GW}$, estimated levels of sEng and PLGF could discriminate pregnant women vulnerable for development of PE. Statistical analyses defined high serum sEng levels estimated at the $12^{\text {th }} \mathrm{GW}$ as the significant early predictor for upcoming PE. Maternal obesity and old age are also related to PE severity and must be considered for prediction.
\end{abstract}

Key Words: Early prediction, endoglin, placental growth factor, preeclampsia

Received: 02 May 2021, Accepted: 12 May 2021

Corresponding Author: Ali A. Bendary, Department of Obstetrics and Gynecology, Faculty of Medicine, Benha University, Egypt, Tel.: 01090650463, E-mail: bendarya264@gmail.com

ISSN: 2090-7265, May 2021, Vol.11, No. 2

\section{INTRODUCTION}

Branched vascular network is crucial to development of the placenta through the regulation of blood vessel growth $^{[1]}$ and depends on secretion of multiple angiogenic factors especially the vascular endothelial growth factor, the placental growth factor, soluble fms-like tyrosine kinase-1 (s-Flt1) and soluble endoglin (sEng) ${ }^{[2]}$.

Preeclampsia (PE) is a complication of pregnancy characterized by gestational hypertension, proteinuria and/or end organ disease ${ }^{[3]}$ with considerable neonatal and maternal morbidities and mortalities ${ }^{[4]}$. Abnormal placentation leads to the release of soluble antiangiogenic factors in line with increased oxidative stress and inflammation ${ }^{[5]}$, these events induce systemic endothelial dysfunction, which in turn causes the clinical manifestations of PE. This mechanism is one of the generally accepted mechanisms for pathogenesis of $\mathrm{PE}^{[4]}$. Alternatively, placental mal-adaptations causes defective or inadequate placentation, apoptosis of invasive cytotrophoblasts ${ }^{[6]}$ inadequate expansive remodeling of the spiral arteries, reduced uteroplacental perfusion pressure ${ }^{[7]}$ and placental ischemia that promotes the release of bioactive factors into the maternal circulation ${ }^{[6]}$.

Endoglin (CD105) is type-I transmembrane glycoprotein, which is highly expressed on proliferating vascular endothelial cells and is an endothelial-specific transforming growth factor- $\beta$ co-receptor ${ }^{[8]}$. Endoglin (Eng) is expressed as a $180-\mathrm{kDa}$ membrane-bound homodimer formed by disulfide-linked monomers from a gene that was localized to chromosome $9 \mathrm{q} 34 \mathrm{ter}^{[9]}$. Eng is responsible for adequate angiogenesis and vascular remodeling as evidenced by animal studies that proved embryonic death due to defective angiogenesis in animal lacking gene for Eng ${ }^{[10]}$, also Eng gene was found to be mutated in patients with hereditary hemorrhagic telangiectasia type $1^{[11]}$ and 
endoglin plays a major role in tumoral and nontumoral adult angiogenesis ${ }^{[12]}$.

Soluble endoglin (sEng) is the circulating form of Eng that was produced by proteolytic processing of membrane Eng ${ }^{[13]}$. Hypoxia and oxidative stress increases membrane bound Eng expression and release of $\mathrm{sEng}^{[14]}$. Soluble Eng has antiangiogenic properties and was found to be overexpressed in patients suffering from hypercholesterolemia, diabetes mellitus and hypertension $^{[15]}$. Moreover, increased levels of sEng correlate with poor outcome in human cancer ${ }^{[16]}$ and sEng was proposed as a marker of endothelial damage ${ }^{[15]}$.

Hypothesis : Serum levels of angiogenic factors had different predictive value for early detection of women vulnerable to develop PE.

\section{AIM OF WORK}

Evaluation of the predictive ability of serum sEng and PLGF levels estimated prior to the $12^{\text {th }}$ gestational week (GW) for discrimination of women liable to develop PE among a wide population of pregnant women.

\section{MATERIAL AND METHODS}

This prospective selective comparative study was conducted at Departments of Obstetrics and Gynecology and Medical Biochemistry, Faculty of Medicine, Benha University in conjunction with multiple private centers, Egypt

The current study was conducted according to the conditions of the Local Ethical Committee since June 2018. The study targets to collect blood samples from all primigravida attending the antenatal care (ANC) units before the $12^{\text {th }} \mathrm{GW}$ and these women were clinically evaluated for fulfilling the inclusion criteria. Inclusion criteria included pregnancy with singleton fetus, no history of renal, hepatic or cardiac diseases, no present or family history of essential hypertension, and no current diabetes mellitus. Clinical evaluation had focused on determination of baseline systolic (SBP) and diastolic blood pressure (DBP) measures and protein level in a voided random urine sample. Proteinuria was determined using urinary dipsticks with a reading at $\geq 1+$ was considered as the cutoff point for diagnosis. Women had proteinuria, but were free of hypertensive history of manifestations were investigated for urinary tract pathology. Thereafter, complete gynecological examination was performed and gestational age (GA) was calculated as the time had lapsed since the $1^{\text {st }}$ day of the last menstrual period and was confirmed by crown-rump length measurement on US examination.

All women fulfilling the inclusion criteria were considered as the study population and were asked to follow-up their BP weekly and attend the outpatient clinic if the BP measure was higher than the baseline reading by $15 \mathrm{mmHg}$ in DBP and/or $30 \mathrm{mmHg}$ in SBP. On arrival to the clinic, BP was measured two times 4-hr apart with the woman at supine position, and if BP measures were persistently higher than baseline measures on the two measurements, the pregnant woman was considered as gestational hypertensive and random urine sample was examined for proteinuria.

\section{Diagnosis and categorization of preeclampsia (PE)}

Preeclampsia (PE) was defined according to the American Society of Hypertension ${ }^{[17]}$ as development of gestational hypertension in a previously normotensive pregnant woman and is associated with proteinuria ${ }^{[18]}$. Regarding severity, PE was categorized as mild and severe according to BP measures obtained during follow-up visits, $\mathrm{PE}$ is considered severe if $\mathrm{SBP}$ is $\geq 160 \mathrm{mmHg}$ or DBP is $\geq 110 \mathrm{mmHg}$ or at least one of the following clinical symptoms occurred: renal insufficiency, pulmonary edema, microvascular disease, thrombocytopenia, impaired liver function, and peripheral severe organ involvement (visual impairment and headache) ${ }^{[19]}$. Concerning timing of development of PE in relation to gestational age, PE was considered of early-onset (EOPE) if diagnosed prior to $34 \mathrm{GW}$ and late-onset (LOPE) if diagnosed after the $34^{\text {th }}$ $\mathrm{GW}^{[20,21]}$

\section{Grouping}

All women who developed PE, irrespective of severity or time of being preeclamptic were collected as PE group and a similar number of women who completed their pregnancy course free of hypertensive manifestations with cross-matched age and body mass index at baseline was collected as Control group.

\section{Blood sampling}

A blood sample of 5-ml was withdrawn under complete aseptic conditions at the start of the $12^{\text {th }} \mathrm{GW}$ for assigned investigations. Blood was allowed to clot and then centrifuged at $3000 \mathrm{rpm}$ for 10 minutes to separate serum that was collected in sterile Eppindorff tube and stores at $-80^{\circ} \mathrm{C}$ till be assayed. Blood samples were collected and numbered by an assistant who was blinded about diagnosis.

\section{Laboratory investigations}

Serum levels of soluble endoglin (sEng) and placental growth factor (Pl-GF) were measured using enzyme linked immunosorbent assay (ELISA) kits according to the manufacturer's instructions and were read using a 96 well microplate ELISA reader (Dynatech. MR 7000).

Human Soluble Endoglin (sENG / sCD105) was measured with the enzyme linked immunoassay (ELISA) kit (Kit-ADR87333.1; catalogue no. MBS269385, MyBioSource Inc., sunny Southern California, San Diego, USA) by quantitative sandwich enzyme immunoassay technique ${ }^{[22]}$

Human placental growth factor (PLGF) was measured with the enzyme linked immunoassay (ELISA) kit (catalogue no. DPG00 SPG00 PDPG00, R\&D systems Inc., Minneapolis, USA) by quantitative sandwich enzyme immunoassay technique ${ }^{[23]}$. 
Study outcomes : Primary outcome is the ability of markers' levels estimated at the $12^{\text {th }} \mathrm{GW}$ to predict women who will develop PE throughout their pregnancy course.

Secondary outcome is the relation between age, BMI, serum levels of studied biomarkers and the extent of change in both SBP and DBP $(\triangle \mathrm{SBP} \& \triangle \mathrm{DBP}$ and equals $\mathrm{BP}$ measure at time of diagnosis of $\mathrm{PE}$ minus the baseline $\mathrm{BP}$ estimated at $1^{\text {st }}$ antenatal visit).

\section{STATISTICAL ANALYSIS}

Obtained data were presented as mean, standard deviation, numbers and percentages. Results were analyzed using One-way Anova for analysis of variance between groups, paired t-test for analysis within each group and Chi-square test ( $\mathrm{X}^{2}$ test) for analysis of non-numeric data. Sensitivity \& specificity of studied parameters as predictors were evaluated using the receiver operating characteristic (ROC) curve analysis judged by the area under the curve (AUC) compared versus the null hypothesis that $\mathrm{AUC}=0.05$ and paired-sample AUC differences was determined versus the null hypothesis that $\mathrm{AUC}=0$. Regression analysis (Stepwise method) was used for stratification of studied parameters as specific predictors. Statistical analysis was conducted using the SPSS (Version 15, 2006) for Windows statistical package. $P$ value $<0.05$ was considered statistically significant.

\section{RESULTS}

Throughout the study duration 102 PE women were diagnosed; 29 women (28.4\%) developed EOPE and 73 women $(71.6 \%)$ had LOPE and 18 women $(17.6 \%)$ had severe, while 84 women $(82.4 \%)$ had mild PE. Mean age $(25.3 \pm 3.9$ years $)$ and BMI $\left(27 \pm 2.9 \mathrm{~kg} / \mathrm{m}^{2}\right)$ of women developed PE were non-significantly $(\mathrm{p}=0.367 \& 0.205$, respectively) higher in comparison to mean age ( $24.8 \pm 4.3$ years) and BMI $\left(26.5 \pm 2.4 \mathrm{~kg} / \mathrm{m}^{2}\right)$ of control women.

Mean at enrolment BP measures showed nonsignificant differences between studied women. However, at time of PE diagnosis, mean BP measures were increased in all studied women with non-significant difference in comparison to their respective baseline $\mathrm{BP}$ measures in control women, while in women who had BP measures diagnostic of PE, BP measures were significantly higher in comparison to their measures obtained at enrolment and to the corresponding measures of control women. Differentially, BP measures at time of PE diagnosis were significantly higher in women developed EOPE and severe $\mathrm{PE}$ in comparison to measures of women had LOPE and mild PE, respectively (Table 1).

Serum levels of sEng estimated at the $12^{\text {th }} \mathrm{GW}$ were significantly higher, while serum PLGF levels were significantly lower in women who developed PE in comparison to control women. Also, serum levels of sEng were significantly higher, while serum PLGF levels were significantly lower in women developed EOPE and severe $\mathrm{PE}$ in comparison to women developed LOPE and mild PE, respectively (Table 2).

Table 1: Blood pressure data of studied women of both groups

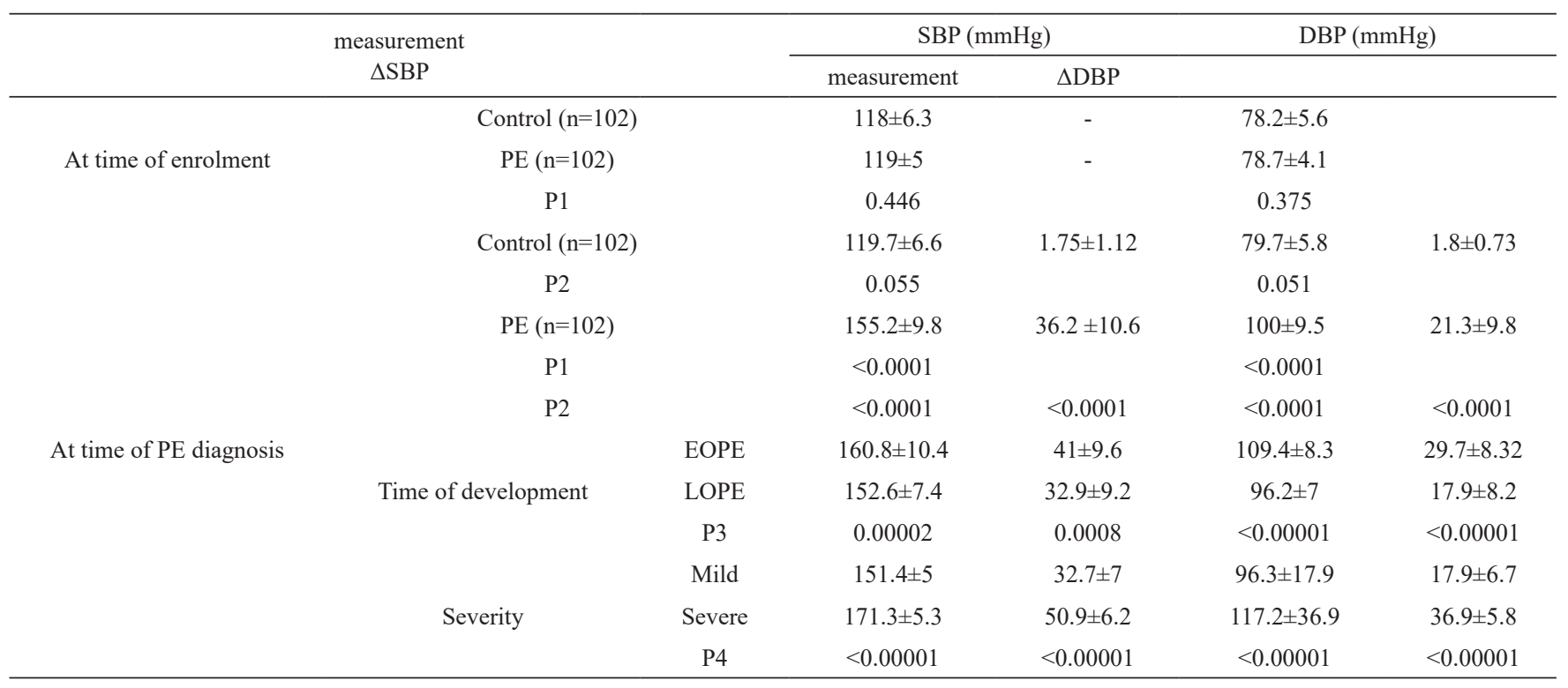

Data are presented as mean, standard deviation; SBP: Systolic blood pressure; DBP: Diastolic blood pressure; $\Delta$ : the extent of change of BP; PE: Pre-eclampsia; P1: significance of difference between PE and control women; P2: significance of difference between BP measures at time of PE diagnosis and time of enrolment; P3: significance of difference between BP measures of women developed EOPE and LOPE; P4: significance of difference between BP measures of women developed mild and severe PE; $p<0.05$ : indicates significant difference; $p>0.05$ : indicates non-significant difference 
Table 2: Serum sEng and PLGF levels estimated at the $12^{\text {th }} \mathrm{GW}$ in studied women

\begin{tabular}{|c|c|c|c|}
\hline & & sEng (ng/ml) & PLGF (pg/ml) \\
\hline & Control $(\mathrm{n}=102)$ & $1.45 \pm 0.39$ & $457.9 \pm 84.8$ \\
\hline \multirow[t]{3}{*}{ Total study women } & $\operatorname{PE}(n=102)$ & $3.3 \pm 0.74$ & $292.2 \pm 82.6$ \\
\hline & P1 value & $<0.00001$ & $<0.00001$ \\
\hline & EOPE & $3.63 \pm 0.83$ & $236.6 \pm 80.7$ \\
\hline \multirow[t]{3}{*}{ Time of development } & LOPE & $3.08 \pm 0.58$ & $314.3 \pm 75.3$ \\
\hline & $\mathrm{P} 2$ value & 0.00024 & 0.00001 \\
\hline & Mild & $3.13 \pm 0.62$ & $313.8 \pm 70.2$ \\
\hline \multirow[t]{2}{*}{ Severity } & Severe & $4.11 \pm 0.73$ & $191.4 \pm 58.7$ \\
\hline & $\mathrm{P} 2$ value & $<0.00001$ & $<0.00001$ \\
\hline
\end{tabular}

Data are presented as mean, standard deviation; sEng: soluble endoglin; PLGF: Placental growth factor; PE: Pre-eclampsia; EOPE: Earlyonset PE; LOPE: Late-onset PE; P1: significance of difference between PE and control women; P2: significance of difference between women developed EOPE and LOPE; P3: significance of difference between women developed mild and severe PE; $p<0.05$ : indicates significant difference; $p>0.05$ : indicates non-significant difference

Correlation analysis of age, and at enrolment BMI and serum levels of sEng and PLGF and development of PE during pregnancy course, irrespective of timing and severity, showed a positive significant correlation (Rho: 0.866, $P<0.0001$ ) between development of PE and serum sEng levels, while the correlation with serum PLGF levels was negative significant (Rho: -0.718 , $P<0.0001)$. On contrary, the correlation with age and BMI was non-significant (Rho: $0.066 \& 0.075, P>0.05$, respectively). Correlation analysis also showed a positive significant correlation between severity of $\mathrm{PE}$ as judged by $\triangle$ SBP with BMI $(\mathrm{r}=0.352, P<0.001)$ and at enrolment serum levels of sEng $(\mathrm{r}=0.612, P<0.001)$, while showed negative significant correlation with at enrolment SBP $\quad(\mathrm{r}=-0.372, \quad P<0.001)$ and serum levels of PLGF $\quad(\mathrm{r}=-0.459, \quad P<0.001)$. Moreover, PE severity as judged by $\triangle \mathrm{DBP}$ was negatively correlated with at enrolment DBP $(\mathrm{r}=-0.410, P<0.001)$ and serum PLGF levels ( $\mathrm{r}=-0.481, P<0.001)$, while was positively correlated with serum sEng levels $(\mathrm{r}=0.604, P<0.001)$.

ROC curve analysis defined $\triangle \mathrm{SBP}$ by $33 \mathrm{mmHg}$ as the significant cutoff point for diagnosis of $\mathrm{PE}$ and analysis of at enrolment SBP measurements, age, BMI and serum levels of sEng and PLGF for prediction of $\triangle$ SBP by 33 $\mathrm{mmHg}$ defined high serum sEng as the significant sensitive predictor for development of PE with increased SBP by $33 \mathrm{mmHg}$. For assurance of the ability of estimated serum sEng as early predictor for development of $\mathrm{PE}$, paired-sample area difference under the ROC curves showed significant differences between AUC for sEng versus that for age, BMI and serum PLGF (Table 3, Figure 1).

Table 3: ROC curve analysis and paired-sample area differences for at enrolment variables as early predictors for development of PE during pregnancy course

\begin{tabular}{|c|c|c|c|c|c|c|c|}
\hline \multicolumn{8}{|c|}{ ROC analysis } \\
\hline \multicolumn{2}{|r|}{ Variable } & $\mathrm{BP}$ & Age & BMI & \multicolumn{2}{|l|}{ Serum sEng } & Serum PLGF \\
\hline \multirow{3}{*}{$\triangle \mathrm{SBP}$} & AUC $( \pm \mathrm{SE})$ & $0.540 \pm 0.127$ & $0.680 \pm 0.158$ & $0.698 \pm 0.152$ & $0.203 \pm 0.093$ & \multicolumn{2}{|c|}{$0.336 \pm 0.134$} \\
\hline & $\mathrm{P} 1$ value & 0.762 & 0.175 & 0.137 & 0.026 & \multicolumn{2}{|c|}{0.218} \\
\hline & $95 \% \mathrm{CI}$ & $0.292 ; 0.789$ & $0.370 ; 0.991$ & $0.400 ; 0.995$ & $0.022 ; 0.385$ & \multicolumn{2}{|c|}{$0.073 ; 0.599$} \\
\hline \multirow{3}{*}{$\triangle \mathrm{DBP}$} & AUC $( \pm$ SE) & $0.869 \pm 0.082$ & $0.812 \pm 0.061$ & $0.579 \pm 0.146$ & $0.199 \pm 0.145$ & \multicolumn{2}{|c|}{$0.782 \pm 0.079$} \\
\hline & P1 value & 0.011 & 0.019 & 0.551 & 0.024 & \multicolumn{2}{|c|}{0.034} \\
\hline & $95 \% \mathrm{CI}$ & $0.679 ; 0.999$ & $0.693 ; 0.931$ & $0.292 ; 0.866$ & $0.0 ; 0.483$ & \multicolumn{2}{|c|}{$0.627 ; 0.938$} \\
\hline \multicolumn{8}{|c|}{ Paired-sample area difference under the ROC curves for at enrolment versus AUC for serum sEng } \\
\hline \multicolumn{2}{|r|}{ Variable } & \multicolumn{2}{|c|}{$\mathrm{BP}$} & Age & \multicolumn{2}{|c|}{ BMI } & Serum PLGF \\
\hline \multirow[t]{4}{*}{$\triangle \mathrm{SBP}$} & AUC difference $( \pm \mathrm{SE})$ & \multicolumn{2}{|c|}{$0.337 \pm 0.486$} & $0.477 \pm 0.522$ & \multicolumn{2}{|c|}{$0.495 \pm 0.508$} & $0.133 \pm 0.506$ \\
\hline & $Z$ value & \multicolumn{2}{|c|}{2.175} & 2.517 & 3.091 & 1.687 & \\
\hline & $\mathrm{P} 2$ value & & & 0.012 & 0.002 & 0.049 & \\
\hline & $95 \% \mathrm{CI}$ & 0.03 & & $0.106 ; 0.849$ & $0.181 ; 0.809$ & $0.513 ; 0.247$ & \\
\hline$\triangle \mathrm{DBP}$ & AUC difference $( \pm \mathrm{SE})$ & & 398 & $0.613 \pm 0.477$ & & - & $0.584 \pm 0.517$ \\
\hline & $Z$ value & & & 3.434 & - & 2.546 & \\
\hline & P2 value & & & 0.001 & - & 0.011 & \\
\hline & $95 \% \mathrm{CI}$ & $0.2^{\prime}$ & & $0.263 ; 0.963$ & - & $1.033 ; 0.134$ & \\
\hline
\end{tabular}

$\Delta$ : Extent of change; BP: Blood pressure; BMI: Body mass index; sEng: Soluble endoglin; PLGF: Placental growth factors; AUC: Area under curve; CI: Confidence interval; P1 indicates significance of AUC versus null hypothesis that $A U C=0.5$; P2 indicates significance of AUC versus null hypothesis that AUC $=0 ; P<0.05$ indicates significant difference; $P>0.05$ indicates non-significant difference 


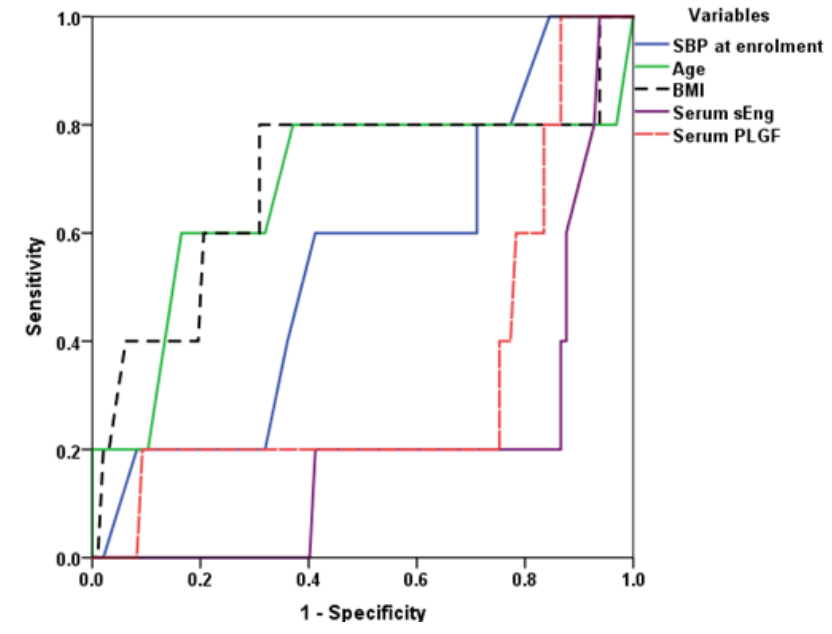

Fig. 1: ROC curve analysis of studied variables as predictors for $\triangle \mathrm{SBP}$ of $33 \mathrm{mmHg}$ among women who developed PE

Considering $\triangle \mathrm{DBP}$ as judgment parameter for development of PE, ROC curve analysis defined $\triangle \mathrm{DBP}$ by $10 \mathrm{mmHg}$ as a diagnostic cutoff point for $\mathrm{PE}$ and excluded BMI as predictor for this $\triangle \mathrm{DBP}$, while assured that age, and serum sEng and PLGF are significant predictors for elevated DBP by $10 \mathrm{mmHg}$ above at enrolment measurement. However, paired-sample area difference under the ROC curves showed significant differences between AUC for sEng versus that for age, DBP and serum PLGF (Table 3, Figure 2).

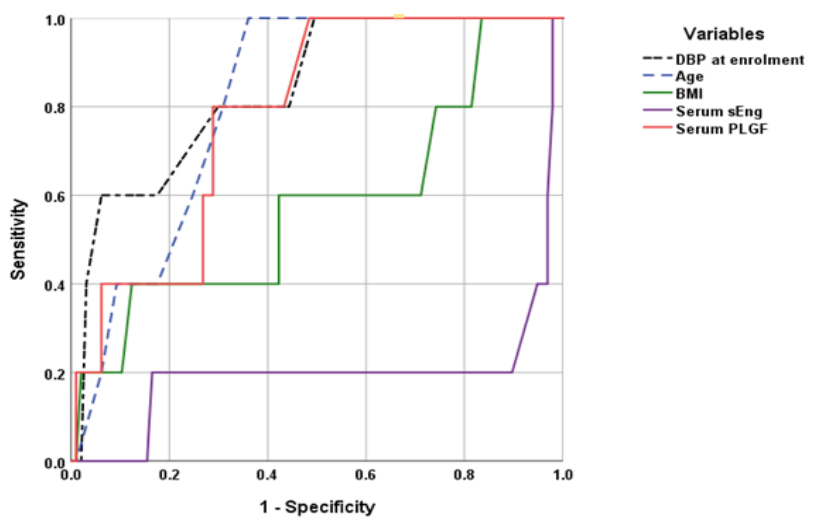

Fig. 2: ROC curve analysis of studied variables as predictors for $\triangle \mathrm{DBP}$ of $10 \mathrm{mmHg}$ among women who developed PE

Considering $\triangle \mathrm{DBP}$ as judgment parameter for development of PE, ROC curve analysis defined $\triangle \mathrm{DBP}$ by $10 \mathrm{mmHg}$ as a diagnostic cutoff point for $\mathrm{PE}$ and excluded BMI as predictor for this $\triangle \mathrm{DBP}$, while assured that age, and serum sEng and PLGF are significant predictors for elevated DBP by $10 \mathrm{mmHg}$ above at enrolment measurement. However, paired-sample area difference under the ROC curves showed significant differences between AUC for sEng versus that for age, DBP and serum PLGF (Table 3, Figure 2).

These findings were assured by Regression analysis for these variables which defined high serum sEng at time of enrolment as a significant early predictor for the possibility of getting elevated both SBP $(\beta=0.612, p<0.001)$ and $\operatorname{DBP}(\beta=0.604, p<0.001)$.

\section{DISCUSSION}

The current study detected high serum sEng and low serum PLGF levels estimated at the $12^{\text {th }} \mathrm{GW}$ in samples of women who had developed PE later during their pregnancy course in comparison to samples of women who completed their pregnancy free of manifestations of PE. Statistical analyses showed a significant correlation between the extent of change in blood pressure $(\triangle B P)$ measures and serum levels of both markers, and ROC curve analysis defined elevated serum sEng at the $12^{\text {th }} \mathrm{GW}$ as the significant predictor for high $\Delta \mathrm{BP}$. These findings indicated the feasibility of using biomarkers as early predictors to discriminate pregnant women vulnerable to hypertensive events during pregnancy and estimation of serum levels of sEng can be used as a sole predictor and its level could predict the severity of PE. These results and assumption are in accordance with multiple recent studies evaluated various markers for prediction of vulnerability for developing PE during pregnancy ${ }^{[2,25,26]}$.

Concerning the diagnostic and predictive ability of estimated serum levels of sEng, the obtained results coincided with Kumer et al. ${ }^{[27]}$ who reported significantly higher serum sEng in women had PE than in control women and Jääskeläinen et al. ${ }^{[28]}$ who also found primiparous pregnancies have more anti-angiogenic profile with higher concentrations of s-Flt1, endoglin and higher sFlt/PlGF ratio during $2^{\text {nd }} / 3^{\text {rd }}$ trimester than multiparous pregnancies. Recently, Sharabi-Nov et al. ${ }^{[29]}$ detected significantly higher serum sEng in women with PE, IUGR and PE+IUGR compared to unaffected controls.

In support of the intimate relation between serum sEng levels and hypertension, therapeutic trials proved the efficacy of the chronic use of perindopril acting through reduction of serum sEng for treatment of chronic hypertensives in comparison to other antihypertensives ${ }^{[30]}$. Also, therapeutic trial using sulfasalazine for preeclamptic women resulted in control of BP with reduction of sFlt-1 and sEng secretion and improvement of endothelial function with upregulation of PLGF ${ }^{[31]}$.

Despite of the high diagnostic ability of high serum sEng, other variable can predict the development of upcoming PE, where low serum PLGF levels could be used as a sole biomarker or in combination with sEng if possible. In line with this data, Giardini et al. ${ }^{[32]}$ obtained results that support the use of PLGF as a biochemical marker for diagnosis of both PE and placental dysfunction, and found normal PLGF is related with a positive pregnancy outcome. Also, Tsviban et al. ${ }^{[33]}$ documented incorporating blood testing of PLGF into the evaluation triage of pregnant women to detect women vulnerable to $\mathrm{PE}$ for its high efficacy and negative predictive value. Moreover, Fillion et al. ${ }^{[34]}$ reported that PLGF, s-Flt1, and their ratio could predict and diagnose early- and intermediate-onset PE but are not useful for LOPE.

Also, BMI determined at the $12^{\text {th }} \mathrm{GW}$ could predict development of PE and was correlated with 
$\triangle \mathrm{BP}$, especially $\triangle \mathrm{SBP}$, while maternal age was correlated with $\triangle \mathrm{DBP}$. These findings go in hand with Lewandowska et $a l^{[35]}$ found the adjusted risk for gestational hypertension, gestational diabetes mellitus, cesarean section, and low birth weight as well as macrosomia $>4000 \mathrm{~g}$ is high in old pregnant women and in obese ones these outcomes are more intense and had also reported that high BMI in the pre-pregnancy period is one of the most likely factor to increase the probability of developing hypertension in pregnancy ${ }^{[36]}$. Moreover, Kawakita et al.$^{[37]}$ detected that higher interpregnancy BMI gain by more than $2 \mathrm{~kg} / \mathrm{m}^{2}$ was associated with increased odds of any hypertensive disorder.

\section{CONCLUSION}

Disturbed angiogenesis milieu with decreased levels of PLGF and increased that of sEng could induce placental dysfunction with subsequent development of PE. At $12^{\text {th }} \mathrm{GW}$, estimated levels of these biomarkers could discriminate pregnant women vulnerable for upcoming development of PE. Statistical analyses defined high serum sEng levels estimated at the $12^{\text {th }} \mathrm{GW}$ as the significant early predictor for upcoming PE. Maternal obesity and old age are also related to PE severity and must be considered for prediction.

\section{CONFLICT OF INTERESTS}

There are no conflict of interests.

\section{REFERENCES}

1. Wu K, Liu F, Wu W, Chen Y, Zhang W. Bioinformatics approach reveals the critical role of TGF- $\beta$ signaling pathway in pre-eclampsia development. Eur J Obstet Gynecol Reprod Biol. 2019 Sep; 240:130-138. Doi: 10.1016/j.ejogrb.2019.06.034.

2. Umapathy A, Chamley L, James J. Reconciling the distinct roles of angiogenic/anti-angiogenic factors in the placenta and maternal circulation of normal and pathological pregnancies. Angiogenesis. 2020 May; 23(2):105-117. Doi: 10.1007/s10456-019-09694-w.

3. Morton J, Levasseur J, Ganguly E, Quon A, Kirschenman R, Dyck J, et al. Characterisation of the Selective Reduced Uteroplacental Perfusion (sRUPP) Model of Preeclampsia. Sci Rep. 2019 Jul 2; 9(1):9565. Doi: 10.1038/s41598-019-45959-6.

4. Smith DD, Costantine MM. The role of statins in the prevention of preeclampsia. Am J Obstet Gynecol. 2020 Aug 17; S0002-9378(20)30868-1. doi: 10.1016/j. ajog.2020.08.040

5. Ali Z, Khaliq S, Zaki S, Ahmad H, Lone K. Altered expression of vascular endothelial growth factor, vascular endothelial growth factor receptor-1, vascular endothelial growth factor receptor-2, and Soluble
Fms-like Tyrosine Kinase-1 in peripheral blood mononuclear cells from normal and preeclamptic pregnancies. Chin J Physiol. May-Jun 2019; 62(3):117122. Doi: 10.4103/CJP.CJP_15_19.

6. Qu H, Khalil R. Vascular mechanisms and molecular targets in hypertensive pregnancy and preeclampsia. Am J Physiol Heart Circ Physiol. 2020 Sep 1; 319(3):H661-H681. Doi: 10.1152/ ajpheart.00202.2020.

7. Govender N, Moodley J, Naicker T. The Use of Soluble FMS-like Tyrosine Kinase 1/Placental Growth Factor Ratio in the Clinical Management of Pre-eclampsia Afr J Reprod Health. 2018 Dec; 22(4):135-143. Doi: 10.29063/ajrh2018/v22i1.14.

8. Pan C, Bloodworth J, Mythreye K, Lee N. Endoglin inhibits ERK-induced c-Myc and cyclin D1 expression to impede endothelial cell proliferation. Biochem Biophys Res Commun. 2012 Aug 3; 424(3):620-3. Doi: 10.1016/j.bbrc.2012.06.163.

9. Campbell L, Yatsenko S, Hixson P, Reimschisel $\mathrm{T}$, Thomas M, Wilson W, et al. Novel 9q34.11 gene deletions encompassing combinations of four Mendelian disease genes: STXBP1, SPTAN1, ENG, and TOR1A. Genet Med. 2012 Oct; 14(10):868-76. Doi: $10.1038 /$ gim.2012.65.

10. Gore B, Izikki M, Mercier O, Dewachter L, Fadel $\mathrm{E}$, Humbert $\mathrm{M}$, et al. Key role of the endothelial TGF- $\beta$ /ALK1/endoglin signaling pathway in humans and rodents pulmonary hypertension. PLoS One. 2014 Jun 23; 9(6):e100310. Doi: 10.1371/journal. pone. 0100310 .

11. Villa D, Cinnante, C Valcamonica G, Manenti G, Lanfranconi S, Colombi A, et al. Hereditary hemorrhagic telangiectasia associated with cortical development malformation due to a start loss mutation in ENG. BMC Neurol. 2020 Aug 26;20(1):316. doi: 10.1186/s12883-020-01890-2.

12. Nogués A, Gallardo-Vara E, Zafra M, Mate P, Marijuan $\mathrm{J}$, Alonso A, et al. Endoglin (CD105) and VEGF as potential angiogenic and dissemination markers for colorectal cancer. World J Surg Oncol. 2020 May 20; 18(1):99. Doi: 10.1186/s12957-020-01871-2.

13. Gallardo-Vara E, Gamella-Pozuelo L, Perez-Roque L, Bartha J, Garcia-Palmero I, Casal J, et al. Potential Role of Circulating Endoglin in Hypertension via the Upregulated Expression of BMP4. Cells. 2020 Apr 16; 9(4):988. Doi: 10.3390/cells9040988. 
14. Oujo B, Perez-Barriocanal F, Bernabeu C, LopezNovoa J. Membrane and soluble forms of endoglin in preeclampsia. Curr Mol Med. 2013 Sep; 13(8):134557. Doi: 10.2174/15665240113139990058.

15. Buda V, Andor M, Baibata D, Cozlac R, Radu G, Coricovac D, et al. Decreased sEng plasma levels in hypertensive patients with endothelial dysfunction under chronic treatment with Perindopril. Drug Des Devel Ther. 2019 Jun 4; 13:1915-1925. doi: 10.2147/ DDDT.S186378.

16. Castillo G, Sánchez-Blanco E, Martín-Villar E, Valbuena-Diez A, Langa C, Pérez-Gómez E, et al. Soluble endoglin antagonizes Met signaling in spindle carcinoma cells. Carcinogenesis. 2015 Feb; 36(2):21222. Doi: $10.1093 /$ carcin/bgu240.

17. Bernhard KA, Siddiqui DS, Leonard KM, Chauhan SP: American college of obstetricians and gynecologists practice bulletins: ascertaining their citation, influence, and utilization. Am J Perinatol. 2014; 31(5):373-82.

18. Lindheimer MD, Taler SJ, Cunningham FG; American Society of Hypertension: ASH position paper. Hypertension in pregnancy. J Clin Hypertens (Greenwich). 2009; 11(4):214-25.

19. Tranquilli AL, Brown MA, Zeeman GG, Dekker G, Sibai BM. The definition of severe and early-onset preeclampsia. Statements from the International Society for the Study of Hypertension in Pregnancy (ISSHP). Pregnancy Hypertens. 2013 Jan; 3(1):44-7.

20. Von Dadelszen P, Magee LA, Roberts JM. Sub classification of preeclampsia. Hypertens Pregnancy. $2003 ; 22: 143-148$.

21. Xu Z, Jin X, Cai W, Zhou M, Shao P, Yang Z, et al. Proteomics Analysis Reveals Abnormal Electron Transport and Excessive Oxidative Stress Cause Mitochondrial Dysfunction in Placental Tissues of Early-Onset Preeclampsia. Proteomics Clin Appl. 2018; 12(5): e1700165.

22. Staff AC, Braekke K, Johnsen GM, Karumanchi SA, Harsem NK. Circulating concentrations of soluble endoglin (CD105) in fetal and maternal serum and in amniotic fluid in preeclampsia. Am J Obstet Gynecol. 2007; 197(2): 176.e1-6.

23. Rebecca T, Braekke K, Harsem NK, Hyer M, Hoover $\mathrm{RN}$, Staff AC. Blood pressure augmentation and maternal circulating concentrations of angiogenic factors at delivery in preeclamptic and uncomplicated pregnancies." Am J Obstet Gynecol., 2008; 199(6): 653.
24. Boutin A, Guerby P, Gasse C, Tapp S, Bujold E: Pregnancy outcomes in nulliparous women with positive first-trimester preterm preeclampsia screening test: the Great Obstetrical Syndromes cohort study. Am J Obstet Gynecol. 2020 Aug; S00029378(20)30836-X. Doi: 10.1016/j.ajog.2020.08.008.

25. Yin Y, Liu M, Yu H, Zhang J, Zhou R: Circulating microRNAs as biomarkers for diagnosis and prediction of preeclampsia: A systematic review and metaanalysis. Eur J Obstet Gynecol Reprod Biol. 2020 Oct; 253:121-132. Doi: 10.1016/j.ejogrb.2020.08.016.

26. Liu Y, Huang J, Yu N, Wei S, Liu Z: Involvement of WNT2 in trophoblast cell behavior in preeclampsia development. Cell Cycle 2020 Sep; 19(17):22072215. Doi: 10.1080/15384101.2020.1802913.

27. Kumer K, Premru-Sršen T, Fabjan-Vodušek V, Tul N, Fabjan T, Osredkar J: Peripheral arterial tonometry and angiogenic biomarkers in preeclampsia. Hypertens Pregnancy. 2018 Nov; 37(4):197-203. Doi: 10.1080/10641955.2018.1524478.

28. Jääskeläinen T, Heinonen S, Hämäläinen E, Pulkki K, Romppanen J, Laivuori $\mathrm{H}$, et al. Angiogenic profile in the Finnish Genetics of Pre-Eclampsia Consortium (FINNPEC) cohort. Pregnancy Hypertens. 2018 Oct; 14:252-259. Doi: 10.1016/j.preghy.2018.03.004.

29. Sharabi-Nov A, Kumar K, Vodušek V, Sršen T, Tul N, Fabjan T, et al. Establishing a Differential Marker Profile for Pregnancy Complications Near Delivery. Fetal Diagn Ther. 2020 June; 47(6):471-484. Doi: $10.1159 / 000502177$.

30. Buda V, Andor M, Baibata D, Cozlac R, Radu G, Coricovac D, et al. Decreased sEng plasma levels in hypertensive patients with endothelial dysfunction under chronic treatment with Perindopril. Drug Des Devel Ther. 2019 June; 13:1915-1925. Doi: 10.2147/ DDDT.S186378.

31. Brownfoot F, Hannan N, Cannon P, Nguyen V, Hastie $\mathrm{R}$, Parry L, et al. Sulfasalazine reduces placental secretion of antiangiogenic factors, up-regulates the secretion of placental growth factor and rescues endothelial dysfunction. EBioMedicine. 2019 Mar; 41:636-648. Doi: 10.1016/j.ebiom.2019.02.013.

32. Giardini V, Rovelli R, Algeri P, Giunti L, Lazzarin $\mathrm{S}$, Callegari $\mathrm{C}$, et al. Placental growth factor as a predictive marker of preeclampsia - PREBIO study - PREeclampsia BIOchemical study. J Matern Fetal Neonatal Med. 2020 Jul 20; 1-7. Doi: 10.1080/14767058.2020.1792878. 
33. Tsviban A, Maymon R, Meiri H, Wiener Y: Using maternal serum placental growth factor (PLGF) for ruling out preeclampsia near deliver: Preliminary results and economic implications in Israel. Harefuah. 2020 Jul; 159(7):492-497.

34. Fillion A, Guerby $\mathrm{P}$, Lachance S, Comeau M, Bussières $\mathrm{M}$, Doucet-Gingras $\mathrm{F}$, et al. Placental Growth Factor and Soluble, Fms-Like Tyrosine Kinase-1 in Preeclampsia: A Case-Cohort (PEARL) Study. J Obstet Gynaecol Can. 2020 Apr; S17012163(20)30341-8. Doi: 10.1016/j.jogc.2020.03.024.

35. Lewandowska M, Sajdak S, Więckowska B, Manevska $\mathrm{N}$, Lubiński J: The Influence of Maternal BMI on
Adverse Pregnancy Outcomes in Older Women. Nutrients. 2020 Sep; 12(9):E2838. Doi: 10.3390/ nu12092838.

36. Lewandowska M, Więckowska B, Sajdak S, Lubiński $\mathrm{J}$ : Pre-Pregnancy Obesity vs. Other Risk Factors in Probability Models of Preeclampsia and Gestational Hypertension. Nutrients. 2020 Sep; 12(9): E2681. Doi: 10.3390/nu12092681.

37. Kawakita T, Downs S, Franco S, Ghofranian A, Thomas A: Interpregnancy body mass index change and risk of hypertensive disorders in pregnancy. J Matern Fetal Neonatal Med. 2020 Sep; 1-6. Doi: 10.1080/14767058.2020.1817371. 